\title{
Modification of Limonoid Metabolism in Suspension Cell Culture of Citrus
}

\author{
Tomoko ENDO ${ }^{1 *}$, Masayuki, KITA ${ }^{1}$, Takehiko SHIMADA ${ }^{1}$, Takaya MORIGUCHI ${ }^{1}$, Tetsushi HIDAKA ${ }^{1}$ \\ Ryoji MATSUMOTO' ${ }^{1}$, Shin HASEGAWA ${ }^{2}$ and Mitsuo OMURA ${ }^{\prime}$ \\ 'Department of Citrus Research, National Institute of Fruit Tree Science, National Agricultural Research \\ Organization, Okitsu, Shimizu, Shizuoka 424-0292, Japan \\ ${ }^{2}$ Western Regional Research Center, Agricultural Research Service, U.S. Department of Agriculture, \\ Buchanan Street, Albany, CA 94710, USA \\ *Corresponding author E-mail address: tomoen@affrc.go.jp
}

Received 8 July 2002; accepted 10 September 2002

\begin{abstract}
For the metabolic engineering of bio-functional substances in Citrus, metabolic changes of limonoids were investigated in embryogenic cell cultures of Citrus and Fortunella. When embryogenic cells of Citrus and Fortunella were cultured for four weeks in the liquid subculture medium containing nomilin, most species converted nomilin to obacunone and limonin. In addition, calamin group limonoids were produced in Fortunella and Calamondin cells. Deacetylnomilin was produced from nomilin in the cells of $C$. ichangensis and its related species. Exogenously added limonin into medium was not metabolized. The embryoid formation in C.aurantium did not affect the limonoid metabolism. All cells cultured without the presence of nomilin or limonin did not generate any limonoids. Furthermore, transgenic $C$. sinensis cell harboring a chimeric limonoid UDP-glucosyltransferase (limonoid GTase) gene was generated. The conversion of limonin into limonin $17-\beta-\mathrm{D}$-glucopyranoside (LG) demonstrated an efficient assay system for the transgene.
\end{abstract}

Key words: bitterness, Citrus, limonoid, limonoid GTase, metabolic engineering.

\section{Abbreviations}

DMSO, dimethylsulfoxide; GTase, UDP-glucosyltransferase; LG, limonin $17-\beta-\mathrm{D}$-glucopyranoside.

\section{Introduction}

Recently, it has been shown that citrus fruit is rich in bio-functional and health-promoting substances such as polymethoxylated flavones (Kawai et al., 2000), auraptene (Ogawa et al., 2000), $\beta$-cryptoxanthin (Ikoma et al., 2001). Among such chemical constituents, there is a group of triterpenoid present in Rutaceae known as limonoids. Limonoids are the cause of delayed-bitterness in juice because intensely bitter limonoids, such as limonin, are gradually developed from non-bitter precursors during the process of juice production. In addition, they have been shown to possess biological functions such as anti-insect feeding (Bentley et al., 1990) and anti-cancer activities (Lam and Hasegawa, 1989; Tanaka et al., 2000). Metabolic engineering of citrus limonoids is of great interest in agricultural, food processing and medical fields. There are wide derivatives in limonoids among Rutaceae family plants. Major citrus species accumulate limonin (Fig. 1III), obacunone (II), nomilin (I) and deacetylnomilin (IV) (Hasegawa and Herman, 1985, 1986; Herman and Hasegawa, 1985). Citrus ichangensis and the relatives accumulate ichangensin (IX, keto and X, ketal) (Benett et al., 1988; Herman et al., 1989; Berhow et al., 1994). Fortunella and related species accumulate calamin group limonids such as calamin (VII) and cyclocalamin (VIII) (Benett and Hasegawa, 1981; Herman et al., 1987).

Radioactive tracer work demonstrated the biosynthesis of limonoids from acetate or mevalonate in the phloem region of stem (Hasegawa et al., 1984, 1986; Ou et al., 1988). Seeds and fruit tissues are capable of biosynthesizing other limonoids starting from nomilin. Limonoid aglycones are endogenously converted to tasteless limonoid glucosides during fruit maturation (Hasegawa et al., 1991; Fong et al., 1992, 1993). This natural debittering process is catalyzed by the enzyme UDP-D-gluco- 


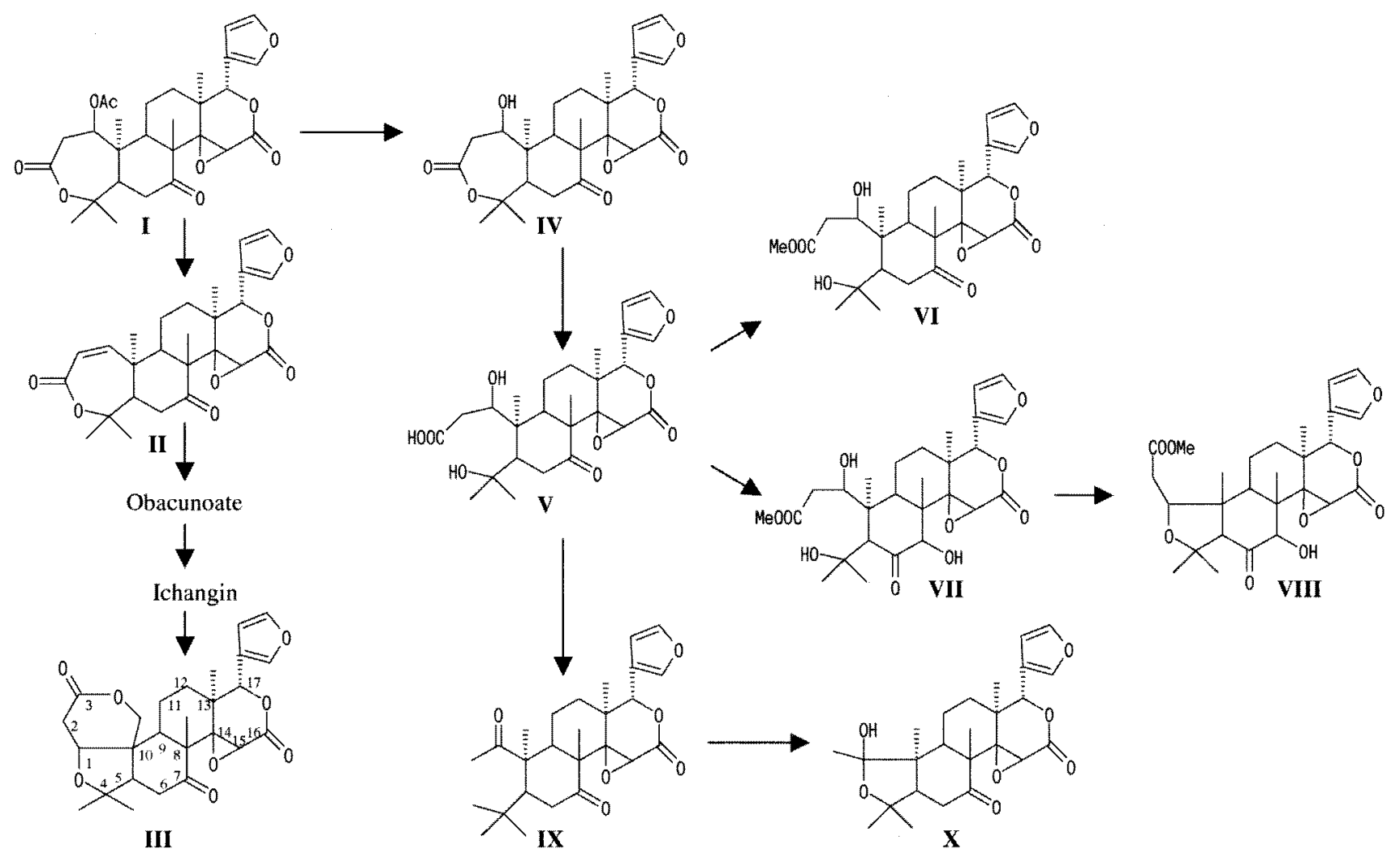

Fig. 1 Limonoids in citrus.

I: Nomilin, II: Obacunone, III: Limonin, IV: Deacetylnomilin, V: Deacetylnomilinate, VI: Methyl deacetylnomilinate, VII: Calamin, VIII: Cyclocalamin, IX: Ichangensin (keto), and $\mathrm{X}$ : Ichangensin (ketal).

side: limonoid glucosyltransferase (limonoid GTase) (Hasegawa et al., 1997). Recently, CitLGT, a gene encoding for a limonoid GTase, was isolated from C. unshiu Marc. (Kita et al., 2000) and could be used as an important tool to manipulate citrus limonoid metabolism (Omura et al., 2000). A plant regeneration system has also been developed for the purpose of genetic engineering. Embryos can be obtained from embryogenic callus by change of culture medium (Hidaka and Omura, 1989). Embryogenic calli can be transformed by an Agrobactrium - mediated method (Hidaka et al., 1990).

Genetic engineering of Citrus to maximize the formation of limonoid glucosides, thereby reducing limonoid bitterness, could be a target for Citrus breeding (Omura et al., 2000). However, it takes long years to evaluate whether such transgenic Citrus actually altered the content of limonoid glucosides in fruits because of the long juvenile duration in woody plants. Therefore, an adequate assay system, such as in calli or young seedlings to evaluate such metabolism has been desired.

However, there has been limited information on limonoid metabolism in such callus cultures in Citrus. Here, we investigated the conversion of limonoids added into medium by citrus embryo- genic cells. By using this system in cultured cells, metabolic alteration by constitutively expressed CitLGT was investigated.

\section{Materials and Methods}

\section{Callus culture}

Embryogenic calli were produced from young or immature nucellar embryos of Citrus aurantium L., C. tangerina (cv. Dancy tangerin), C. reticulata Blanco (cv. Ohta ponkan), C. sinensis Osbeck (cvs. Trovita orange, Washington navel, Valencia orange), C. ichangensis Swingle, C. junos hort. Ex Tanaka, C. hystrix DC., C. madurensis Lour., Fortunella crassifolia Swingle, and $F$. hindsii Swingle. They were induced on the Gelrite-solidified medium consisted with Murashige and Skoog's basal components (Murashige and Skoog, 1962) supplemented with $0.2 \mathrm{M}$ sucrose and $50 \mu \mathrm{M}$ kinetin $(\mathrm{pH}$ 5.6). Cultures were maintained by subculturing at 2 - month intervals under $16 \mathrm{~h}$ illumination regime at $25^{\circ} \mathrm{C}$ for 5 to 10 years as previously described (Hidaka and Omura, 1989). Prior to experiments, calli were suspended in the liquid medium with the same components unless otherwise mentioned. 
Addition of limonoids into culture medium

For the assay of limonoid conversion, approximately $1 \mathrm{~g}$ calli were suspended in the $200 \mathrm{ml}$ liquid medium. Limonin and nomilin were dissolved with dimethylsulfoxide (DMSO) and added into liquid medium to final concentration of $0.01 \%(\mathrm{w} / \mathrm{v})$. Equal amount of DMSO was added to the medium of the controls. Suspension cultures were orvitary shaken at $120 \mathrm{rpm}$ under $16 \mathrm{~h}$ illumination regime at $25^{\circ} \mathrm{C}$ for 4 weeks. To investigate the limonoid metabolism in callus during embryogenesis, suspension cells of $C$. aurantium were placed on the nylon membrane filter $(22 \mu \mathrm{m}$ pore size), under which 3 sheets of filter paper were layered and supplied culture medium to the cells. The medium for embryogenesis was consisted of MS basal components with $0.1 \mathrm{M}$ sorbitol and $0.1 \mathrm{M}$ galactose (Hidaka and Omura, 1989). The medium for callus proliferation containing $0.2 \mathrm{M}$ sucrose and $50 \mu \mathrm{M}$ kinetin was also examined. Both cultures were under $16 \mathrm{~h}$ illumination regime at $25^{\circ} \mathrm{C}$ for 4 weeks.

\section{Analysis of limonoids}

After culturing for 4 weeks, the cells were collected on nylon mesh by filtration and washed with water 3 times to remove the culture medium. They were dried in the oven at $65^{\circ} \mathrm{C}$ for 3 days and used for analysis. One gram of dried sample was grounded in $5 \mathrm{ml}$ of $0.5 \mathrm{M}$ Tris- $\mathrm{HCl}$ buffer $(\mathrm{pH} 8.0)$ with a Polytron homogenizer. The mixture was then filtered through celite and the filtrate was treated with a $\mathrm{C}-18$ Sep-Pak. Limonoids were analyzed by TLC and HPLC (Herman et al., 1989) or LC-MS (Manners et al., 2000). In the representative sample from C. sinensis (cv. Trovita orange), the quantities of limonoids were determined on the chromatographic areas with two independently replicated analyses.

\section{Transformation of callus with CitLGT}

Plasmid for transformation was generated as follows. Full length of CitLGT cDNA (Kita et al., 2000) was excised with $X b a \mathrm{I}$ and $K p n \mathrm{I}$. A binary vector pBE2113 (Mitsuhara et al., 1996) was digested with $X b a \mathrm{I}$ and $S a c I$, and ligated $X b a \mathrm{I}-\mathrm{SacI}$ linker fragment from pUC18 (Yanisch-Perron et al., 1985). Resultant vector plasmid was digested with $X b a \mathrm{I}$ and $K p n I$, and ligated with the CitLGT fragment. This construct was incorporated into Agrobacterium tumefaciens strain LBA4404 by triparent mating. Agrobacterium infection of Washington navel callus was carried out according to the method of Hidaka et al. (1990) with a slight modification. About $1 \mathrm{~g}$ of callus was suspended in $40 \mathrm{ml}$ liquid medium for transformation. During 3 days co-cultivation, calli were plated on the nylon membrane filter, same as mentioned above, and washed with MS liquid medium everyday.

Transformed calli were selected on the Gelritesolidified medium consisted with MS basal components supplemented with $0.2 \mathrm{M}$ sucrose, $50 \mu \mathrm{M}$ kinetin, $50 \mathrm{mg} \mathrm{l}^{-1}$ geneticin and $250 \mathrm{mg} \mathrm{l}^{-1}$ Claforan (pH 5.6). Gene incorporation was confirmed by standard PCR amplification technique with a primer set of $35 \mathrm{~S}$ promoter $\left(5^{\prime}-\mathrm{ATCTCCACTGACG}\right.$ TAAGGGATGACG-3') and $L G T$ - GR (5' - TCAATACTGTACACGTGTCCGTCG-3') sequences. Thermal condition was 30 cycles of 1 min at $94{ }^{\circ} \mathrm{C}$, $1 \mathrm{~min}$ at $58^{\circ} \mathrm{C}$ and $1 \mathrm{~min}$ at $72^{\circ} \mathrm{C}$.

Accumulation of CitLGT transcript was analyzed by RT-PCR with the 1st strand cDNA as a template. Total RNA isolation and 1st strand cDNA synthesis from transformed calli were performed by the methods described previously (Kita et al., 2000). A set of primers used for RT-PCR amplification was $L G T-G F$ (5'-ATGGGAACTGAATCTCTTGTTCAT-3') and $L G T$-GR. Thermal condition was same with that of PCR.

\section{Results and Discussion}

\section{Limonid metabolisms in suspension cell culture}

In order to investigate the metabolism of limonoids in embryogenic callus cells, nomilin or limonin was added into liquid medium, and limonoid contents of cultured cells were analyzed. As shown in Table 1 and the HPLC-chromatogram (Fig. 2), a

Table 1. Limonoids detected in the callus culture added with nomilin.

\begin{tabular}{|c|c|c|c|c|c|}
\hline \multirow[b]{2}{*}{ Cell clone } & \multicolumn{5}{|c|}{ Limonoid } \\
\hline & $I^{1)}$ & IV & II & III & $\begin{array}{l}\text { calamin } \\
\text { group }^{2 y}\end{array}$ \\
\hline \multicolumn{6}{|l|}{ C. sinensis } \\
\hline cv. Washington navel & + & - & + & + & - \\
\hline cv. Trovita orange & + & - & + & + & - \\
\hline cv. Valencia orange & + & - & + & + & - \\
\hline C. tangerina & + & - & + & + & - \\
\hline \multicolumn{6}{|l|}{ C. reticulata } \\
\hline cv, Ohta ponkan & + & - & + & + & - \\
\hline C. junos & + & + & + & + & - \\
\hline C. ichangensis & + & + & + & + & - \\
\hline C. hystrix & + & + & + & + & - \\
\hline C. madurensis & + & + & + & + & + \\
\hline$F$. crassifolia & + & + & + & - & + \\
\hline F. hindsii & + & + & + & - & + \\
\hline
\end{tabular}

\footnotetext{
"See Fig.1

${ }^{2}$ See text.
} 

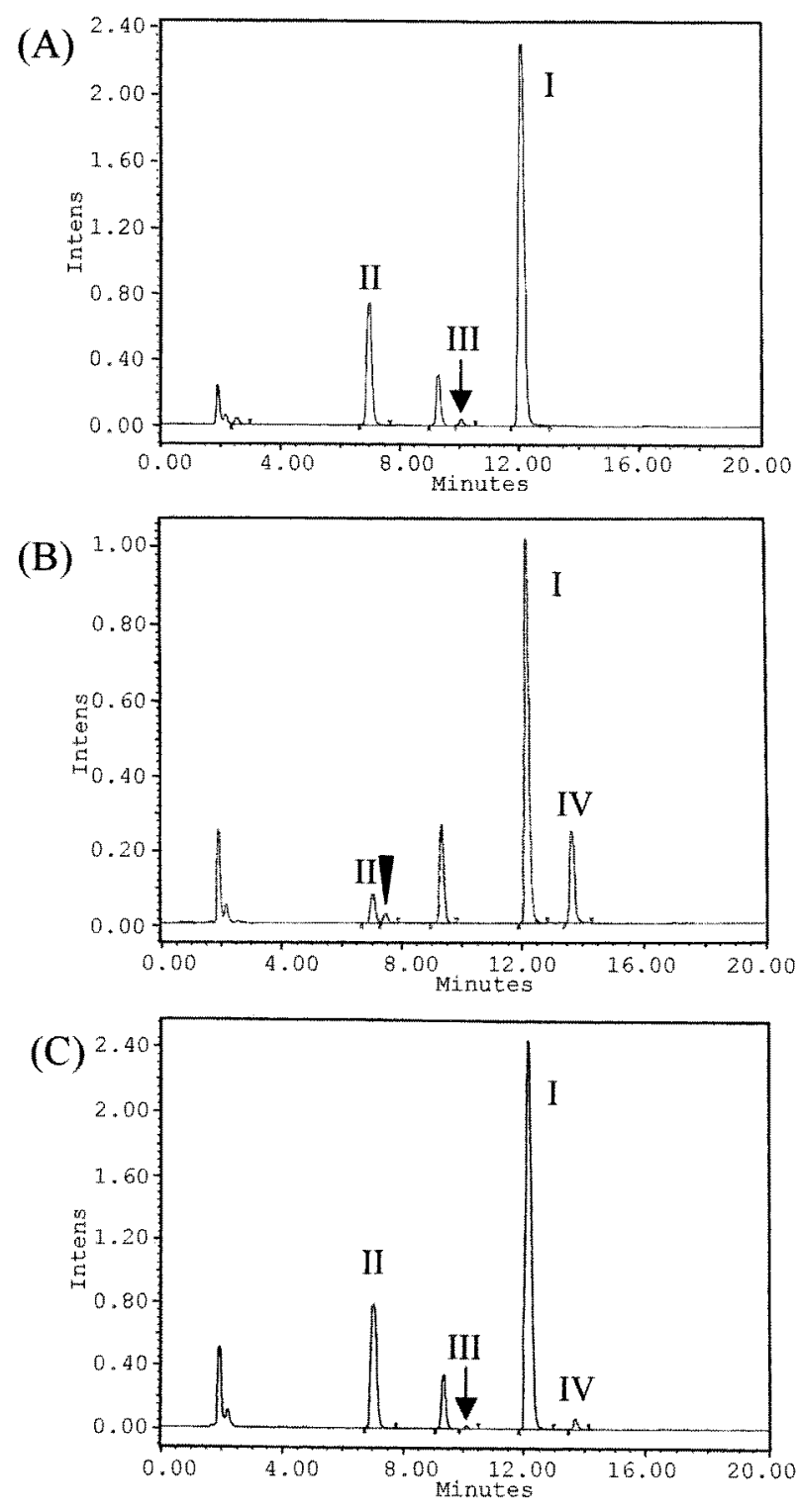

Fig. 2 HPLC profiles of limonoids in suspension cells cultured with nomilin.

I, II, III and IV: See Fig. 1. An arrowhead in (B) points a peak of a mixture composed of methyl deacetylnomilinate and unidentified compound similar to calamin, which is only observed in Fortunella. (A) C. sinensis cv. Trovita orange; (B) F. crassifolia; (C) C. junos.

variety of limonoids were detected over the clearly detectable levels in the cells cultured with nomilin. The cultured cells of sweet oranges (Citrus sinensis cvs. Washington navel, Trovita orange and Valencia orange) and mandarins (C. tangerina and C. reticulata) contained obacunone (II) and limonin (III) along with nomilin (I), corresponding that fruits and seeds of those species contained such major limonoids (Ozaki et al., 1991). The cells of Trovita orange contained $1410 \mathrm{ppm}$ nomilin per $1 \mathrm{~g}$ dried cells. The same cells contained $487 \mathrm{ppm}$ obacunone and $44 \mathrm{ppm}$ limonin, which correspond to $34.5 \%$ and $3.1 \%$ of nomilin, respectively. From the cells of two Fortunella species ( $F$. crassifolia and $F$. hindsii) and Fortunella-related hybrid Calamondin $(C$. madurensis), calamin group limonoids, such as methyl deacetylnomilinate (VI), calamin (VII) and cyclocalamin (VIII), were detected in addition to obacunone. Although limonin was detected in Calamondin cells, two Fortunella species did not produce it at the detectable levels. The suspension cells of $C$. ichangensis, $C$. junos and $C$. hystrix produced obacunone, limonin and deacetylnomilin (IV). However, they did not produce their specific major limonoid, ichangensin (IX, keto and X, ketal). It appears that the conversion from nomilin to deacetylnomilin would be regulated basically in a genotype-specific manner in cultured cells same as intact fruits and seeds, suggesting that nomilinacetyl esterase, which is unique to this species, is active in the cultured cells as well as in various tissues (Herman, et al., 1989). However, enzyme activities involved in dehydroxylation of deacetylnomilinate followed by decarboxylation would be repressed in cultured cells.

All of the suspension cells did not metabolize limonin added to the media (data not shown). Together with the results of nomilin feeding experiment, this indicates that the major pathway from nomilin to limonin via obacunone is unidirectional in cultured cells, which is consistent with previous reports (Herman and Hasegawa, 1985; Hasegawa and Herman, 1986).

Limonoid metabolisms during embryogenesis from callus of C. aurantium

It is generally known that secondary metabolism is repressed in nondifferentiated cells and this repression can be reversed during the course of differentiation as in organogenesis and embryogenesis. Gavish et al. (1989) used embryo cultures of $C$. paradisi in the experiments of biotransformation of flavanones in the cell culture, and showed that the endogenous production of naringin in Citrus cell culture is developmentally regulated. However, there has been a little information for the limonoid metabolism in the cell culture. Limonin contents were rapidly decreased in calli from fruit flavedo, albedo, juice vesicle, leaf, stem and cotyledon of Citrus, but they were recovered in the regenerated shoots from callus (Barthe et al., 1987).

In this study, limonoid metabolisms were investigated in both embryoid-inductive and cell-proliferating conditions (Table 2). Embryogenesis of $C$. aurantium has been induced on the embryogenesis medium. Embryoids with green color were observed after 4 weeks on the same medium, while original 
Table 2. Limonoids of $C$. aurantium cells after 4 weeks culture.

\begin{tabular}{clllll}
\hline \multirow{2}{*}{$\begin{array}{c}\text { Added } \\
\text { Limonid }\end{array}$} & Culture condition & I $^{\text {l) }}$ & IV & II & III \\
\cline { 2 - 5 } Nomilin & Embryogenesis & + & + & + & + \\
& Cell proliferation & + & + & + & + \\
Limonin & Embryogenesis & - & - & - & + \\
& Cell proliferation & - & - & - & + \\
Control & Embryogenesis & - & - & - & - \\
$($ DMSO) & Cell proliferation & - & - & - & - \\
& Before culture & - & - & - & - \\
\hline
\end{tabular}

"See Fig. 1.

callus continued to proliferate on the subculture medium. Before the embryo formation had occurred, no limonoids were detectable in such cultures without feeding of limonoids. When limonin was added to the culture media, only limonin (III) was recovered from the cells on both embryoidinductive and cell-proliferating conditions. In contrast, deacetylnomilin (IV), obacunone (II) and limonin (III) as well as nomilin (I) were detected when nomilin was added into both media, independent from the cell differentiation state. These results showed that there were no differences between cellproliferating and embryoid-inductive conditions in nomilin or limonin biotransformation.

Since neither proliferating callus cells nor embryoids produced limonoids without nomilin addition to the medium, the suppression of limonoid biosynthesis would occur prior to the biosynthesis of nomilin independent from embryogenesis. This corresponds to the report by Hasegawa et al. (1980) in which de novo synthesis of limonoids from acetate in the seeds could not be demonstrated. Since nomilin biosynthesis occurs only in phloem of stem of intact plant (Hasegawa et al, 1986; Ou et al., 1988), further development of young embryoids to plantlets with well-organized vascular system would be required for biosynthesis of limonoids from acetate in vitro.

\section{Limonoid metabolism in CitLGT-introduced cells}

Because the glucosylation of limonoids is a natural debittering process, regulation of biosynthesis of limonoid glucosides is one of major targets for genetic modification to produce Citrus trees that have fruits free of limonoid bitterness (Kita et al., 2000; Omura et al., 2000).

Transgenic cells of $C$. sinensis cv. Washington navel, in which CitLGT encoding UDP-D-glucoside: limonoid glucosyltransferase (Kita et al., 2000) was ectopically expressed, were established via Agrobacterium infection. RT-PCR analysis

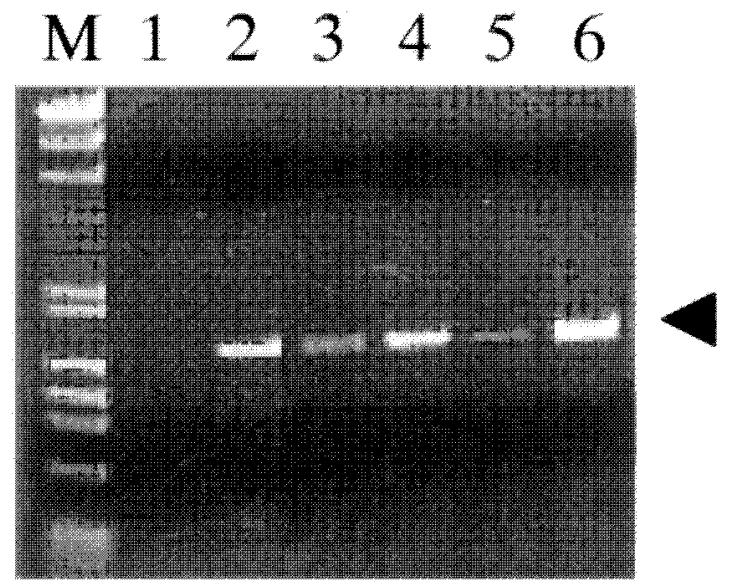

Fig. 3 RT-PCR pattern of Washington navel callus transformed with CitLGT gene.

RT-PCR products were electrophoresed on agarose gel and stained with ethidium bromide. An arrowhead indicates $1536 \mathrm{bp}$ target position of CilLGT gene. M: $\lambda$ HindIII + $\phi$ X174 HaeIII, Lane 1: untransformed callus, Lane 2-6: transgenic callus (Lane 2:4-1, Lane 3:5-1, Lane 4: 6-1, Lane 5: 7-1 and Lane 6:5-10).

indicated that transgenic callus clones were transcriptionally active for CitLGT, whereas control callus was not (Fig. 3). Production of limonoid glucosides was tested in independent transgenic cell lines. In the first experiment, transformed calli of 3 independent clones were cultured and limonin $17-$ $\beta-D$-glucopyranoside (LG) was detected in 2 clones when limonin was added into medium (Table 3). Following experiment using other 5 lines showed that only one line produced LG (data not shown). Limonin was produced from nomilin in each cell line (Table 3), showing metabolism of limonoid aglycones in consistent with the results of non-transformed cells. However, LG was not produced when nomilin was added into medium, even in the cell lines in which LG was produced from limonin. This might be due to the relatively low level contents of limonin produced from nomilin in 
Table 3. Limonids detected in the CitLGTintroduced callus of Washington navel.

\begin{tabular}{clccc}
\hline \multirow{2}{*}{ Cell line } & \multicolumn{1}{c}{$\begin{array}{c}\text { Added } \\
\text { limonoid }\end{array}$} & \multicolumn{3}{c}{ Limonoid } \\
\cline { 3 - 5 } $4-1$ & Control (DMSO) & - & - & - \\
& Nomilin & + & + & - \\
& Limonin & - & + & + \\
$5-1$ & Control (DMSO) & - & - & - \\
& Nomilin & + & + & - \\
& Limonin & - & + & + \\
\multirow{5}{*}{$5-10$} & Control (DMSO) & - & - & - \\
& Nomilin & + & + & - \\
& Limonin & - & + & - \\
\hline
\end{tabular}

"See Fig. 1.

${ }^{2)}$ limonin $17-\beta-D-$ glucopyranoside

cultured cells, as mentioned above, and/or to relatively low activity of limonin GTase derived from the transgene. We also observed that the native limonoid GTase showed very low activity, when it was isolated from fruit tissues (Hasegawa et al., 1997) and when its cDNA was expressed as a fusion protein with GST in E. coli (Kita et al, 2000). In addition, the fact that only a few lines could produce LG from limonin added into medium suggests several factors by which limonoid glucoside accumulation is regulated besides transcript accumulation of CitLGT. However, this is the first finding that the callus cells of Citrus produced limonoid glucosides if transformed with a cDNA encoding the limonoid GTase. Prior to this report, callus cells of Citrus have not been used for the assay system of the bio-functional substances, but our results support that citrus cell culture is a useful tool for the evaluation of limonoid metabolism.

\section{Acknowledgements}

We are indebted to Mrs. S. Yamanashi and Mrs. M. Ikeda for their technical assistance and to Dr. T. Kubo and Dr. A. Breksa for thier helpful advice. This work was supported in part by a project grant from the Japanese Ministry of Agriculture, Forestry and Fisheries. Contribution No. 1272 of the NIFTS.

\section{References}

Barthe, G. A., Jourdan, P. S., McIntosh, C. A., Mansell, R. L., 1987. Naringin and limonin production in callus cultures and regenerated shoots from citrus sp. J. Plant Physiol., 127: 55-65.

Benett, R. D., Hasegawa, S., 1981. Limonoids of calamondin seeds. Tetrahedron, 37: 17-24.

Benett, R. D., Herman, Z., Hasegawa, S., 1988. Ichangensin: a new cilrus limonoid. Phytochemistry, 27: 1.543-1545. Bentley, M. D., Rajab, M. S., Mendel M. J., Alford, A. R, 1990. Limonoid model insect antifeedants. J. Agric. Food Chem., 38: 1400-1403.

Berhow, M. A., Omura, M., Ohta, H., Ozaki, Y., Hasegawa, S., 1994. Limonoids in seeds of three Citrus hybrids related to Citrus ichangensis. Phytochemistry, 36: 923925.

Fong, C. H., Hasegawa, S., Coggins, C. W., Atkin, D. R., Miyake, M., 1992. Contents of limonoids and limonin $17-\beta-D-$ glucopyranoside in fruit tissue of Valencia orange during fruit growth and maturation. J. Agric. Food Chem., 40: 1178-1181.

Fong, C. H., Hasegawa, S., Miyake, M., Ozaki, Y., Coggins, C. W., Atkin, D. R., 1993. Limonoids and their glucosides in Valencia orange seeds during fruit growth and development. J. Agric. Food Chem., 41: 112-115.

Gavish, H., Lewinsohn, E., Vardi, A., Fluhr, R., 1989. Production of flavanone neohesperidosides in citrus embryos. Plant Cell Rep., 8: 391 - 394.

Hasegawa, S., Benett, R. D., Maier, V. P., 1984. Biosynthesis of limonoids in Citrus seedlings. Phytochemistry, 23: 1601-1603.

Hasegawa, S., Benett, R. D., Verdon, C. P., 1980. Limonoids in Citrus seeds: origin and relative concentration. $\mathrm{J}$. Agric. Food Chem. 28: 922-925.

Hasegawa, S., Herman, Z., 1985. Biosynthesis of obacunone from nomilin in Citrus limon. Phytochemistry, 24: 1973 $-1974$.

Hasegawa, S., Herman, Z., 1986. Biosynthesis of limonoids: conversion of deacetylnomilinate to nomilin in Citrus limon. Phytochemistry, 25: 2523-2524.

Hasegawa, S., Herman, Z., Orme, E., Ou, P., 1986. Biosynthesis of limonoids in Citrus: sites and translocation. Phytochemistry, 25: 2783-2785.

Hasegawa, S., Suhayda, C. G., Hsu, W., Robertson, G. H., 1997. Purification of limonoid glucosyltransferase from navel orange albedo tissues. Phytochemistry, 46: 33 37.

Hasegawa, S., Ou, P., Fong, C. H., Herman, Z., Coggins, C. W., Atkin, D. R., 1991. Changes in the limonoate $A$. ring lactone and limonin $17-\beta-D$-glucopyranoside content of navel oranges during fruit growth and maturation. J. Agric. Food Chem., 39: 262-265.

Herman, Z., Benett, R. D., Ou, P., Fong, C. H., Hasegawa, S., 1987. Metabolism of limonoids in the Citrus hybrid Clamondin. Phytochemistry, 26: 2247-2250.

Herman, Z., Hasegawa, S., 1985. Limonin biosynthesis from obacunone via obacunoate in Citrus limon. Phytochemistry, 24: $2911-2912$.

Herman, Z., Hasegawa, S., Fong, C. H., Ou P., 1989. Limonoids in Citrus ichangensis. J. Agric. Food Sci, 37: $850-851$.

Hidaka, T., Omura, M., 1989. Control of embryogenesis in Citrus cell culture. Bull. Fruit Tree Res. Stn. B 16: 117.

Hidaka, T., Omura, M., Ugaki, M., Tomiyama, M., Kato, A., Ohshima, M., Motoyoshi, F., 1990. Agrobacterium mediated transformation and regeneration of Citrus spp. 
From suspension cells. Japan. J. Breed., 40: 199-207.

Ikoma, Y., Komatsu, A., Kita, M., Ogawa, K., Omura, M., Yano, M., Moriguchi, T., 2001. Expression of a phytoene synthase gene and characteristic carotenoid accumulation during citrus fruit development. Pysiol. Plant., 111: $232-238$.

Kawai, S., Tomono, Y., Katase, E., Ogawa, K., Yano, M., Koizumi, M., Ito, C., Furukawa, H., 2000. Quantitative study of flavonoids in leaves of Citrus plants. J. Agric. Food Chem., 48: 3865-3871.

Kita, M., Hirata, Y., Moriguchi, T., Endo-Inagaki, T., Matsumoto, R., Hasegawa, S., Suhayda, C. G., Omura, M., 2000. Molecular cloning and characterization of a novel gene encoding limonoid UDP-glucosyltransferase in Citrus. FEBS Lett., 469: 173-178.

Lam, L. K. T., Hasegawa, S., 1989. Inhibition of Benzo[a]pyrene-induced forestomach neoplasia in mice by citrus limonoids. Nutr. Cancer, 12: 43-47.

Manners, G. D., Hasegawa, S., Bennett, R. D., Wong, R. Y., 2000. LC-MS and NMR techniques for the analysis and characterization of Citrus limonoids. In: Berhow, M. A. et al. (Eds.): Citrus Limonoids Functional Chemicals in Agriculture and Food, pp. 40-59. American Chemical Society, Washington, DC.

Mitsuhara, I., Ugaki, M., Hirochika, H., Ohshima, M., Murakami, T., Gotoh, Y., Katayose, Y., Nakamura, S., Honkura, R., Nishimiya, S., Ueno, K., Mochizuki, A., Tanimoto, H., Tsugawa, H., Otsuki, Y., Ohashi, Y., 1996. Efficient promoter cassettes for enhanced expression of foreign genes in dicotyledonous and monocoty- ledonous plants. Plant Cell Physiol., 37: 49-59.

Murashige, T., Skoog, F, 1962. A revised medium for rapid growth and bioassays with tobacco tissue cultures. Physiol. Plant., 15: 473-497.

Ogawa, K., Kawasaki, A., Yoshida, T., Nesumi, H., Nakano, M., Ikoma, Y., Yano, M., 2000. Evaluation of auraptene content in citrus fruits and their products. J. Agric. Food Chem., 48: 1763-1769.

Omura, M., Kita, M., Endo-Inagaki, T., Moriguchi, T., Matsumoto, R., Suhayda, C., Hasegawa, S., 2000. Genetic evaluation and modification of the accumulation of limonoids in Citrus. In: Berhow, M. A. et al. (Eds.): Citrus Limonoids Functional Chemicals in Agriculture and Food, pp. 230-237. American Chemical Society, Washington, DC.

Ou, P., Hasegawa, S., Herman, Z., Fong, C. H., 1988. Limonoid biosynthesis in the stem of Citrus limon. Phytochemistry, 27: 115-118.

Ozaki, Y., Fong, C. H., Herman, Z., Maeda, H., Miyake, M., Ifuku, Y., Hasegawa, S., 1991. Limonoid glucosides in citrus seeds. Agric. Biol. Chem., 55: 137-141.

Tanaka, T., Kohno, H., Tsukio, Y., Honjo, S., Tanino, M., Miyake, M., Wada, K., 2000. Citrus limonoids obacunone and limonin inhibit azoxymethane--induced colon carcinogenesis in rats. Biofactors, 13: $213-218$.

Yanisch-Perron, C., Vieira, J., Messing, J., 1985. Improved $M-13$ phage cloning vectors and host strains nucleotide sequence of the $\mathrm{M}-13 \mathrm{mp} 18$ and pUC19 vectors. Gene, 33: 103-119. 